\title{
Active Vibration Control of an Axially Translating Robot Arm with Rotating-Prismatic Joint Using Self-Sensing Actuator
}

\author{
Liang Zhao and Zhen-Dong Hu \\ School of Aerospace Engineering and Applied Mechanics, Tongji University, Shanghai 200092, China \\ Correspondence should be addressed to Zhen-Dong Hu; zdhu@mail.tongji.edu.cn
}

Received 7 August 2014; Accepted 6 October 2014

Academic Editor: Mickaël Lallart

Copyright ( 2015 L. Zhao and Z.-D. Hu. This is an open access article distributed under the Creative Commons Attribution License, which permits unrestricted use, distribution, and reproduction in any medium, provided the original work is properly cited.

\begin{abstract}
Active vibration control of an axially translating robot arm with rotating-prismatic joint using self-sensing actuator is investigated. The equations of the system are derived by Lagrange's equation with the assumed mode method. The displacement and velocity control law is used to configure the self-sensing actuator, which provides the active damping and stiffness effect to the structure. The numerical simulations reveal that the tip deflection of the arm can be effectively reduced by the self-sensing actuator. The amplitude of sensor voltage is inversely proportional to the length of axially translating arm. And higher feedback control gain results in lower sensor voltages and vibration amplitudes.
\end{abstract}

\section{Introduction}

Dynamics of elastic beam sliding in a prismatic joint is an important problem in many engineering applications, such as textile machines, automotive belts, band saws, robot arms, and elevator cables. The axial movement of the structures can lead to transverse vibration and will exert great effect on product quality and the safety and reliability of work processes. Therefore, they have received great attention.

Many research papers deal with the beam axially translating through a stationary prismatic joint. Wang and Wei [1] used Newton's approach in deriving the governing equation of an axially moving beam and utilized Galerkin's approximation technique in discretizing the elastic displacements. Stylianou and Tabarrok [2] employed finite element method to analyse an axially moving beam. They made the number of elements fixed while the sizes of the elements change with time. Fung et al. [3] applied Hamilton's principle to derive nonlinear partial differential equations of an axially moving beam with a tip mass. Zhu and Ni [4] studied the axially translating cantilever beam with an arbitrarily varying length. The dynamic stability of the continuous media relative to the inertial and moving coordinate systems is studied from the energy standpoint. Wang et al. $[5,6]$ used Hamilton's principle to investigate the axially translating elastic
Bernoulli-Euler and viscoelastic cantilever beam with timevariant velocity, respectively. Chang et al. [7] investigated the vibration and stability of an axially moving cantilever Rayleigh beam. The finite element method with variabledomain elements is used to derive the equations of motion and two kinds of axial motions including constant-speed extension deployment and back-and-forth periodical motion are considered. Ghaleh and Malaek [8] studied dynamics of deploying arms carrying tip mass by applying assumed modes method and employing the numerical solutions to find the arm responses.

The problem becomes more difficult if the beam is translating through a rotating-prismatic joint. For a translating and rotating beam, the interaction between rigid and flexible body motions is highly demanded. Gaultier and Cleghorn [9] used finite element method to analyze the vibration of a spatially translating and rotating beam to model elastic link manipulators. The internal and external damping effects were also included in the model. Yuh and Young [10] analyzed the dynamics of a beam experiencing a combination of rotational and translational motions. An approximation scheme was developed by using assumed modes method. The validity of the approximate model was evaluated by a series of experimental work. Dynamic response of the elastic beam undergoing various motions was investigated by computer 
simulation. Al-Bedoor and Khulief [11] analyzed the dynamics of an elastic arm sliding in a rotating-prismatic joint by using finite element technique. In the finite element model all the inertia coupling terms were considered. Time dependent boundary conditions were used in order to account for the prismatic joint. The effect of an end mass was also taken into account in the model. And they [12] presented a dynamic model for the vibrational motion of an elastic beam with prismatic and revolute joints. The Lagrangian approach in conjunction with the assumed modes technique is employed in deriving the equations of motion. Basher [13] investigated the dynamic modeling of a single-link flexible beam having both rotational and translation motions and the effects of higher-order dynamics on the response of the beam. An analytical model of the beam, characterized by an infinite number of modes, was developed using Euler-Bernoulli beam equation and modal expansion method. The infinitedimensional transcendental transfer function for the manipulator was formulated without modal approximation that was conveniently transformed into state-space form. Farid and Salimi [14] proposed an inverse dynamic approach to determine the required actuating torque and force for a planar flexible-link manipulator with revolute-prismatic joints such that its end-point follows a given trajectory. The formulation includes all of the nonlinear terms due to large rotation of the links. Kalyoncu [15] investigated the dynamic response of a flexible robot manipulator with rotating-prismatic joint. The tip end of the flexible robot manipulator traces a multistraight-line path under the action of an external driving torque and an axial force.

Recently, piezoelectric materials have been more and more often applied to improve structural behavior. Such materials can actuate forces by electrical excitation, making them suitable as actuators for vibration control. Besides, they can sense deformation and generate a real-time voltage signal. Therefore they can be used as sensor as well. In most cases, a piezoelectric element performs a single transducer function: either sensing or actuation. Sun and Huang [16] developed an analytical formulation for modeling the behavior of laminated composite beams with integrated piezoelectric sensor and actuator. And the analytical solutions for active vibration control and suppression of smart laminated composite beams are presented. Raja et al. [17] derived a coupled finite element procedure to model the piezoelectric actuation in a smart sandwich beam. Chen et al. [18] considered a slender laminated composite beam with piezoelectric layers subjected to axial periodic compressive loads. The top and bottom piezoelectric layers act as actuator and sensor, respectively, and the influence of the feedback control gain on the response of the beam is evaluated. Lin and Nien [19] investigated the modeling and active vibration control of a smart beam using piezoelectric damping-modal actuators/sensors. $\mathrm{Hu}$ and $\mathrm{Ng}$ [20] developed an approach for active vibration control of flexible structures with integrated piezoelectric actuators using a robust vibration control method. Qiu et al. [21] used piezoelectric ceramic patches as sensors and actuators to suppress the vibration of the smart flexible clamped plate. Kumar and Narayanan [22] investigated the optimal placement of collocated piezoelectric

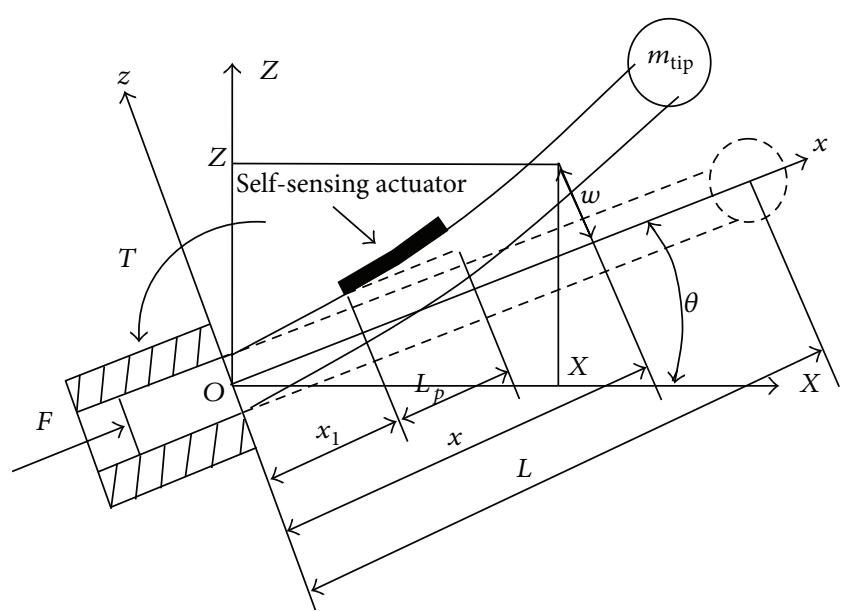

FIGURE 1: An axially translating robot arm with rotating-prismatic joint.

actuator-sensor pairs on flexible beams using a model-based linear quadratic regulator (LQR) controller. Song and Li [23] applied the piezoelectric actuator/sensor pairs to control the vibration of supersonic beams.

The concept of combining sensing and actuating features in the same device leads to the so-called self-sensing techniques [24]. In the self-sensing actuator configuration, the piezoelectric element is used simultaneously as both a sensor and an actuator. A self-sensing based system needs half the number of transducers normally necessary, making this approach appreciable for applications. Yellin and Shen [25] used the self-sensing technique to form a self-sensing active constrained layer damping treatment, which can improve the damping ability of the classical passive constrained layer. Gao and Liao [26] studied the active vibration control of simply supported beams with enhanced self-sensing active constrained layer damping treatments.

In this paper, we aim to study the active vibration control of an axially translating robot arm with rotating-prismatic joint using self-sensing actuator. Lagrange's equation with the assumed mode method is employed to derive the equations of motion. And the displacement and velocity control law is used to configure the self-sensing actuator.

\section{Formulation of the Equations of Motion}

An axially translating robot arm with rotating-prismatic joint is displayed in Figure 1. A tip mass $m_{\text {tip }}$ is considered to be concentrated at the free end of the arm. The flexible arm slides in the prismatic joint under the action of axial force $F$. The sliding motion of the arm is assumed to be frictionless. The initial length of the arm is $L_{0}$ and the variation in the length of the arm is denoted by $S$. Torque $T$ rotates prismatic joint about $Y$-axis and the prismatic joint is assumed to be rigid. The robot arm experiences a combination of rotational and translational gross motion. $X Y Z$ is the global reference frame, while $x y z$ is the rotating and translating reference frame. Angle between the rotating and translating reference frame $x y z$ and the global reference frame $X Y Z$ is denoted by $\theta . \mathrm{d} m$ on the arm denotes an infinitesimal mass as seen in Figure 1. 
The displacement of $\mathrm{d} m$ from the undeformed position in $z$ direction is denoted by $w$. The piezoelectric patch as the selfsensing actuator is bonded on the top surface of the arm and positioned by the coordinates $x_{1}(t)$ and $x_{2}(t)$ in the reference frame $x y z$. The length of the piezoelectric patch is $L_{p}$. It is obvious that when the arm is axially translating, $x_{1}(t)$ and $x_{2}(t)$ change with time and can be expressed as $x_{1}(t)=$ $x_{1}(0)+S$ and $x_{2}(t)=x_{1}(t)+L_{p}$, respectively. In this paper, we assume $0 \leq x_{1}(0)<x_{2}(0) \leq L_{0}$ and $0 \leq x_{1}(t)<x_{2}(t) \leq L(t)$ during the arm axial movement. $h$ and $h_{p}$ are the thicknesses of the arm and piezoelectric patch, respectively. The width of the arm and the piezoelectric patch are both $b$.

Euler-Bernoulli beam theory is employed to analyze the arm. Based on the Kirchhoff assumptions, the normal stress and strain of the arm in the axial direction are expressed as

$$
\begin{aligned}
& \sigma_{x}=E \varepsilon_{x}, \\
& \varepsilon_{x}=-z \frac{\partial^{2} w}{\partial x^{2}},
\end{aligned}
$$

where $E$ is the modulus of elasticity of the arm. Let the piezoelectric patch be polarized along the $z$ direction. The axial stress, strain, and the electrical displacement of the piezoelectric patch can be expressed as

$$
\begin{aligned}
\sigma_{x}^{p} & =c_{11} \varepsilon_{x}^{p}-e_{31} E_{z}, \\
\varepsilon_{x}^{p} & =-z \frac{\partial^{2} w}{\partial x^{2}}, \\
D_{z} & =e_{31} \varepsilon_{x}^{p}+\epsilon_{33} E_{z},
\end{aligned}
$$

where $c_{11}$ is the elastic stiffness, $D_{z}$ is the electrical displacement and $E_{z}=V_{0}(t) / h_{p}$ is the electrical field in the $z$ direction [23], and $V_{0}(t)$ is the external applied voltage. $\epsilon_{33}$ is the dielectric constant and $e_{31}$ is the piezoelectric constant.

The global position of an arbitrary material point $p$ on the arm can be expressed as

$$
R_{p}=A \cdot r_{p},
$$

where $A$ is the rotational transformation matrix from the moving coordinate system $x z$ to the fixed reference frame $X Z$ and $r_{p}$ is the location of the point $p$ in the rotating coordinate system $x z$; they can be written as

$$
\begin{aligned}
& A=\left[\begin{array}{cc}
\cos \theta & -\sin \theta \\
\sin \theta & \cos \theta
\end{array}\right], \\
& r_{p}=\left[\begin{array}{ll}
x & w
\end{array}\right]^{T} .
\end{aligned}
$$

The velocity of an arbitrary material point $p$ is

$$
\dot{R}_{p}=A \cdot \dot{r}_{p}+\dot{\theta} \frac{\mathrm{d} A}{\mathrm{~d} \theta} r_{p},
$$

where $\dot{\theta}$ is the angular velocity of the prismatic joint, and

$$
\dot{r}_{p}=\left[\begin{array}{ll}
\dot{x} & \frac{\mathrm{d} w}{\mathrm{~d} t}
\end{array}\right]^{T}=\left[\begin{array}{ll}
v & \frac{\mathrm{d} w}{\mathrm{~d} t}
\end{array}\right] .
$$

Derivative of $w$ with respect to time is written as

$$
\frac{\mathrm{d} w}{\mathrm{~d} t}=\dot{w}+\dot{x} w^{\prime} .
$$

Substituting (4a), (4b), (6), and (7) into (5), we will arrive at

$$
\dot{R}_{p}=\left[\begin{array}{l}
(\dot{x}-w \dot{\theta}) \cos \theta-\left(x \dot{\theta}+\dot{w}+\dot{x} w^{\prime}\right) \sin \theta \\
(\dot{x}-w \dot{\theta}) \sin \theta+\left(x \dot{\theta}+\dot{w}+\dot{x} w^{\prime}\right) \cos \theta
\end{array}\right] .
$$

The total kinetic energy of the system can be written as

$$
\begin{aligned}
K= & \frac{1}{2} \rho A_{b} \int_{0}^{L} \dot{R}_{p}^{T} \dot{R}_{p} \mathrm{~d} x+\frac{1}{2} \rho_{p} A_{p} \int_{x_{1}}^{x_{1}+L_{p}} \dot{R}_{p}^{T} \dot{R}_{p} \mathrm{~d} x \\
& +\left.\frac{1}{2} m_{\text {tip }} \dot{R}_{p}^{T} \dot{R}_{p}\right|_{x=L}+\frac{1}{2}\left(J_{b}+J_{p}\right) \dot{\theta}^{2},
\end{aligned}
$$

where $\rho$ and $\rho_{p}$ are the mass density of the arm and the piezoelectric patch, $A_{b}=b h$ and $A_{p}=b h_{p}$ are the cross-sectional area of the arm and the piezoelectric patch, respectively, and $J_{b}$ and $J_{p}$ are the rotational inertia of the arm and the piezoelectric patch, respectively. By substituting for the value of $\dot{R}_{p}$ from (8) and making use of some trigonometric properties, the total kinetic energy of the system can be expressed as

$$
\begin{aligned}
K= & \frac{1}{2} \rho A_{b} \int_{0}^{L} \bar{V}^{2} \mathrm{~d} x+\frac{1}{2} \rho_{p} A_{p} \int_{x_{1}}^{x_{1}+L_{p}} \bar{V}^{2} \mathrm{~d} x \\
& +\left.\frac{1}{2} m_{\mathrm{tip}} \bar{V}^{2}\right|_{x=L}+\frac{1}{2}\left(J_{b}+J_{p}\right) \dot{\theta}^{2},
\end{aligned}
$$

where

$$
\bar{V}^{2}=(\dot{x}-w \dot{\theta})^{2}+\left(x \dot{\theta}+\dot{w}+\dot{x} w^{\prime}\right)^{2} .
$$

The strain energy of the arm and the strain energy and electric potential energy of the piezoelectric patch can be written as

$$
\begin{aligned}
U_{s}= & \frac{1}{2} \int_{0}^{L} \int_{-h / 2}^{h / 2} b \sigma_{x} \varepsilon_{x} \mathrm{~d} z \mathrm{~d} x \\
& +\frac{1}{2} \int_{x_{1}}^{x_{1}+L_{p}} \int_{h / 2}^{h / 2+h_{p}} b\left(\sigma_{x}^{p} \varepsilon_{x}^{p}-D_{z} E_{z}\right) \mathrm{d} z \mathrm{~d} x .
\end{aligned}
$$

Substituting (1a), (1b), (2a), (2b), and (2c) into (12), we can obtain

$$
\begin{aligned}
U_{s}= & \frac{1}{2} \int_{0}^{L} E I_{b}\left(w^{\prime \prime}\right)^{2} \mathrm{~d} x \\
& +\frac{1}{2} \int_{x_{1}}^{x_{1}+L_{p}}\left[E I_{p}\left(w^{\prime \prime}\right)^{2}+2 B_{1} V_{0}(t) w^{\prime \prime}-B_{2} V_{0}^{2}(t)\right] \mathrm{d} x,
\end{aligned}
$$

where

$$
\begin{gathered}
E I_{b}=\frac{E b h^{3}}{12}, \quad E I_{p}=\frac{1}{3} c_{11} b\left[\left(\frac{h}{2}+h_{p}\right)^{3}-\left(\frac{h}{2}\right)^{3}\right], \\
B_{1}=\frac{e_{31} b\left(h+h_{p}\right)}{2}, \quad B_{2}=\frac{b \epsilon_{33}}{h_{p}} .
\end{gathered}
$$


The gravitational potential energy for the arm, including the piezoelectric patch and the end mass, can be written as

$$
\begin{aligned}
U_{g}= & \frac{1}{2} \rho A_{b} g L^{2} \sin \theta \\
& +\rho_{p} A_{p} g L_{p}\left(x_{1}+\frac{L_{p}}{2}\right) \sin \theta+m_{\text {tip }} g L \sin \theta .
\end{aligned}
$$

The component of absolute acceleration in $x$ direction can be written as

$$
a_{x}=\ddot{x}-\dot{\theta}^{2} x-\ddot{\theta} w .
$$

And the component of gravitational acceleration in $x$ direction is

$$
g_{x}=-g \sin \theta
$$

Thus the axial inertial force is

$$
F_{x}=\rho A_{b} \int_{x}^{L}\left(-a_{x}+g_{x}\right) \mathrm{d} x+m_{\text {tip }}\left(-\left.a_{x}\right|_{x=L}+g_{x}\right) \text {. }
$$

Because the piezoelectric patch is very thin, axial inertial force of the piezoelectric patch is neglected. Now, the potential energy due to axial shortening can be written as

$$
U_{x}=\frac{1}{2} \int_{0}^{L} F_{x}\left(w^{\prime}\right)^{2} \mathrm{~d} x .
$$

The total potential energy of the system can be expressed as

$$
U=U_{s}+U_{g}+U_{x} .
$$

Lagrange's equation with the assumed mode method is used to determine the equation of motion of the structural system. To use the assumed mode method, the displacements of the arm can be expressed in terms of the generalized coordinates and displacement shape functions. According to $[5,8]$, the transverse displacement is written as

$$
\begin{aligned}
w(x, t) & =\frac{1}{\sqrt{L(t)}} \sum_{i=1}^{n} \phi_{i}(x, t) \cdot q_{i}(t) \\
& =\frac{1}{\sqrt{L(t)}}[\phi] \cdot\{q\}^{T},
\end{aligned}
$$

where $[\phi]=\left[\phi_{1}, \ldots, \phi_{n}\right],\{q\}=\left\{q_{1}, \ldots, q_{n}\right\}$, and

$$
\begin{aligned}
\phi_{i}= & {\left[\cosh \left(\lambda_{i} \frac{x}{L}\right)-\cos \left(\lambda_{i} \frac{x}{L}\right)\right] } \\
& -\frac{\cosh \left(\lambda_{i}\right)+\cos \left(\lambda_{i}\right)}{\sinh \left(\lambda_{i}\right)+\sin \left(\lambda_{i}\right)}\left[\sinh \left(\lambda_{i} \frac{x}{L}\right)-\sin \left(\lambda_{i} \frac{x}{L}\right)\right], \\
i & =1,2, \ldots, n,
\end{aligned}
$$

where $\lambda_{i}$ is obtained from the following characteristic equation:

$$
1+\cosh \left(\lambda_{i}\right) \cos \left(\lambda_{i}\right)=0 \quad i=1,2, \ldots, n .
$$

Lagrange's equation is written as

$$
\frac{\mathrm{d}}{\mathrm{d} t}\left(\frac{\partial \ell}{\partial \dot{q}}\right)-\frac{\partial \ell}{\partial q}=F, \quad \ell=K-U .
$$

Substituting the energy expressions into (24), the equation of motion of the whole structural system is gotten:

$$
[M]\left\{\ddot{q}^{T}\right\}+[C]\left\{\dot{q}^{T}\right\}+[K]\left\{q^{T}\right\}+\{Q\}+\left[F_{V}\right] V_{0}(t)=0,
$$

where

$$
\begin{aligned}
{[M]=} & \rho A_{b} \int_{0}^{L}[\phi]^{T}[\phi] \mathrm{d} x \\
& +\rho_{p} A_{p} \int_{x_{1}}^{x_{1}+L_{p}}[\phi]^{T}[\phi] \mathrm{d} x+\left.m_{\text {tip }}\left([\phi]^{T}[\phi]\right)\right|_{x=L}
\end{aligned}
$$$$
[C]=\rho A_{b} \int_{0}^{L}\left[C_{1}\right] \mathrm{d} x+\rho_{p} A_{p} \int_{x_{1}}^{x_{1}+L_{p}}\left[C_{1}\right] \mathrm{d} x,
$$$$
[K]=E I_{b} \int_{0}^{L} \frac{\mathrm{d}^{2}[\phi]^{T}}{\mathrm{~d} x^{2}} \frac{\mathrm{d}^{2}[\phi]}{\mathrm{d} x^{2}} \mathrm{~d} x
$$$$
+E I_{p} \int_{x_{1}}^{x_{1}+L_{p}} \frac{\mathrm{d}^{2}[\phi]^{T}}{\mathrm{~d} x^{2}} \frac{\mathrm{d}^{2}[\phi]}{\mathrm{d} x^{2}} \mathrm{~d} x
$$$$
+\rho A_{b} \int_{0}^{L}\left[K_{1}\right] \mathrm{d} x+\int_{0}^{L} F_{x} \frac{\mathrm{d}[\phi]^{T}}{\mathrm{~d} x} \frac{\mathrm{d}[\phi]}{\mathrm{d} x} \mathrm{~d} x
$$$$
+\rho_{p} A_{p} \int_{x_{1}}^{x_{1}+L_{p}}\left[K_{1}\right] \mathrm{d} x+\left.m_{\mathrm{tip}}\left[K_{1}\right]\right|_{x=L},
$$

$$
\begin{aligned}
& {[Q]=\rho A_{b} \int_{0}^{L}\left(\left[Q_{1}\right]+\left[Q_{2}\right]\right) d x} \\
& +\rho_{p} A_{p} \int_{x_{1}}^{x_{1}+L_{p}}\left[Q_{1}\right] \mathrm{d} x+\left.m_{\text {tip }}\left[Q_{1}\right]\right|_{x=L} \\
& F_{V}=B_{1} \sqrt{L} \int_{x_{1}}^{x_{1}+L_{p}} \frac{\mathrm{d}^{2}[\phi]^{T}}{\mathrm{~d} x^{2}} \mathrm{~d} x \\
& {\left[C_{1}\right]=\frac{\dot{L}}{L}\left[\left(\frac{\mathrm{d}[\phi]^{T}}{\mathrm{~d} x}[\phi]+[\phi]^{T} \frac{\mathrm{d}[\phi]}{\mathrm{d} x}\right)(L-x)-[\phi]^{T}[\phi]\right] \text {, }} \\
& {\left[K_{1}\right]=\left(\frac{3 \dot{L}^{2}}{4 L^{2}}-\frac{\ddot{L}}{2 L}-\dot{\theta}^{2}\right)[\phi]^{T}[\phi]} \\
& +\left[\frac{\dot{L}^{2}}{2 L^{2}}(9 L+5 x)+\frac{\ddot{L}}{L}(2 L-x)\right][\phi]^{T} \frac{\mathrm{d}[\phi]}{\mathrm{d} x} \\
& -\frac{\dot{L}^{2}}{2 L^{2}}(L-x) \frac{\mathrm{d}[\phi]^{T}}{\mathrm{~d} x}[\phi] \\
& +\frac{\dot{L}^{2}}{L^{2}}(2 L-x)(L-x)[\phi] \frac{\mathrm{d}^{2}[\phi]}{\mathrm{d} x^{2}} \\
& -\frac{\dot{L}^{2}}{L}(2 L-x) \frac{\mathrm{d}[\phi]^{T}}{\mathrm{~d} x} \frac{\mathrm{d}[\phi]}{\mathrm{d} x}, \\
& F_{x}=\rho A_{b}\left[\frac{\dot{\theta}^{2}}{2}\left(L^{2}-x^{2}\right)-(g \sin \theta+\ddot{L})(L-x)\right] \\
& +m_{\text {tip }}\left(\dot{\theta}^{2} L-g \sin \theta-\ddot{L}\right) \text {, }
\end{aligned}
$$




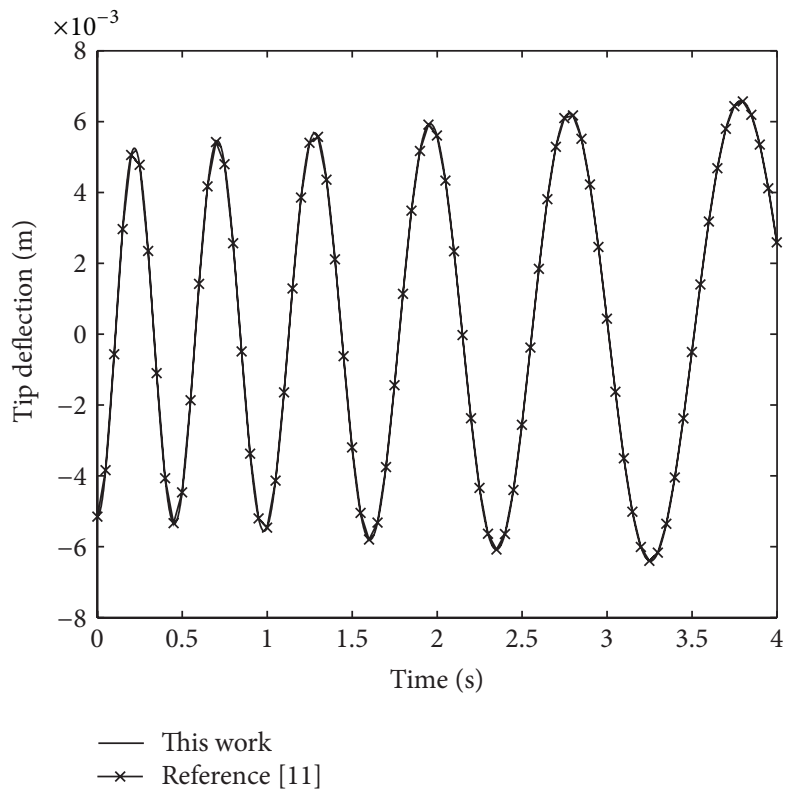

FIgURE 2: Tip deflection of the axially translating beam when $L(t)=1.8+0.3 t$.

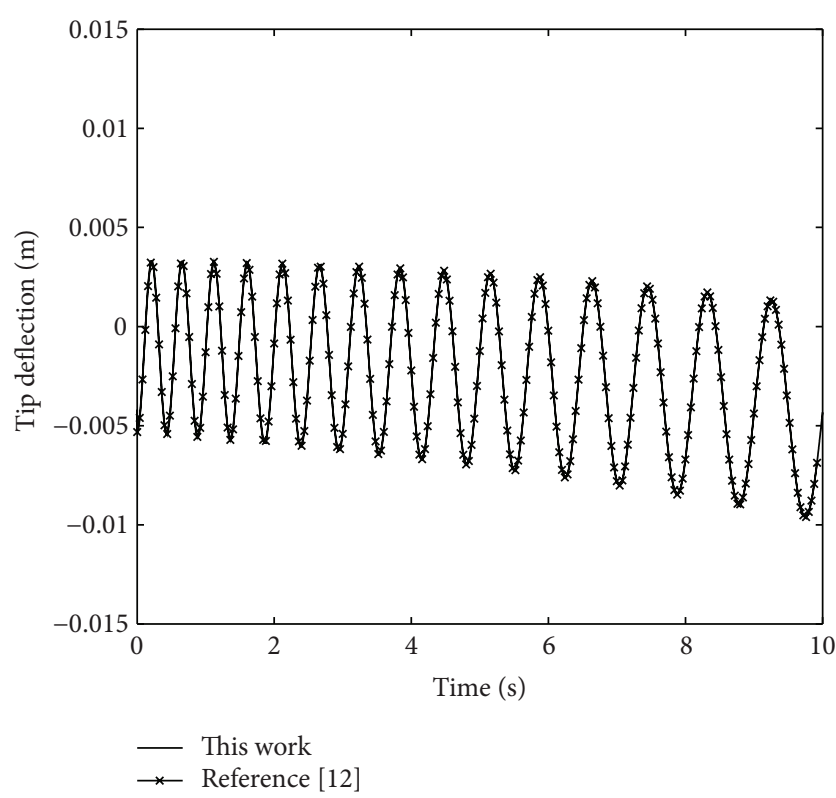

FIGURE 3: Tip deflection of the axially translating and rotating beam when $L(t)=1.8+0.1 t, \dot{\theta}=0.785 \mathrm{rad} / \mathrm{s}$.

$$
\begin{aligned}
{\left[Q_{1}\right]=} & \sqrt{L}\left[(2 \dot{\mathrm{L}} \dot{\theta}+x \ddot{\theta})[\phi]^{T}-\dot{L} \dot{\theta} x \frac{\mathrm{d}[\phi]^{T}}{\mathrm{~d} x}\right], \\
{\left[Q_{2}\right]=} & \frac{3}{2 \sqrt{L}} \ddot{\theta}\left[\rho A_{b} \int_{0}^{L}[\phi] \mathrm{d} x+\left.m_{\text {tip }}[\phi]\right|_{x=L}\right] \\
& \times\left(\{q\} \frac{\mathrm{d}[\phi]^{T}}{\mathrm{~d} x} \frac{\mathrm{d}[\phi]}{\mathrm{d} x}\{q\}^{T}\right) .
\end{aligned}
$$

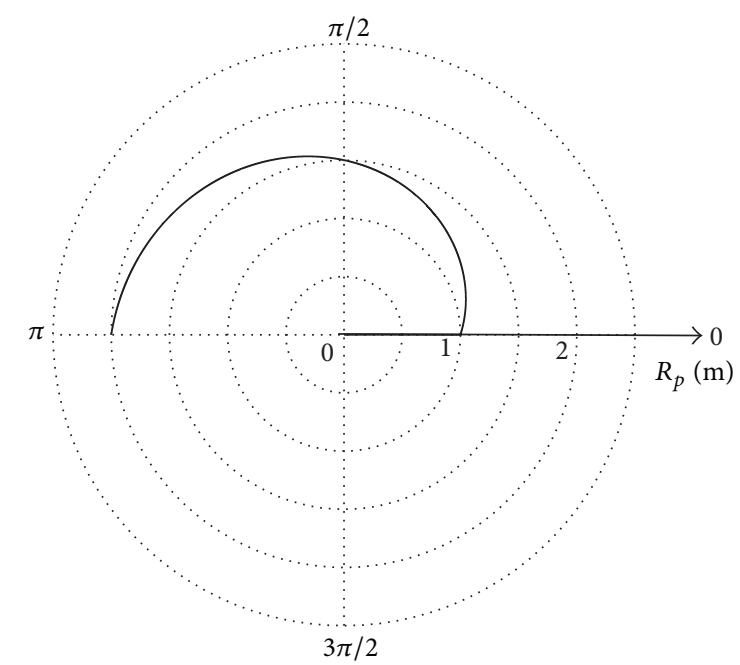

FIgURE 4: Tip trajectory of the arm when $L(t)=1+0.2 t, \dot{\theta}=$ $0.2 \pi \mathrm{rad} / \mathrm{s}$.

Equation (25) characterizes the piezoelectric actuator driven under the external applied voltage $V_{0}(t)$. The matrix $\left[Q_{2}\right]$ represents the nonlinear inertia coupling between the beam reference motion and the local elastic deformations. Clearly, when the prismatic joint rotates with a uniform angular velocity we need to solve a linear problem.

\section{Active Vibration Control}

When the arm moves with deformation, the charge $Q_{s}$ developed by structural deformation on the piezoelectric patch can be found by integrating the electric displacement $D_{z}$ in (2b) over the electrode area $A_{p}[23,26]$ :

$$
Q_{s}(t)=\int_{A_{p}} D_{z} \mathrm{~d} A_{p}=e_{31} b \int_{x_{1}(t)}^{x_{2}(t)}\left(-z_{p} \frac{\partial^{2} w}{\partial x^{2}}\right) \mathrm{d} x,
$$




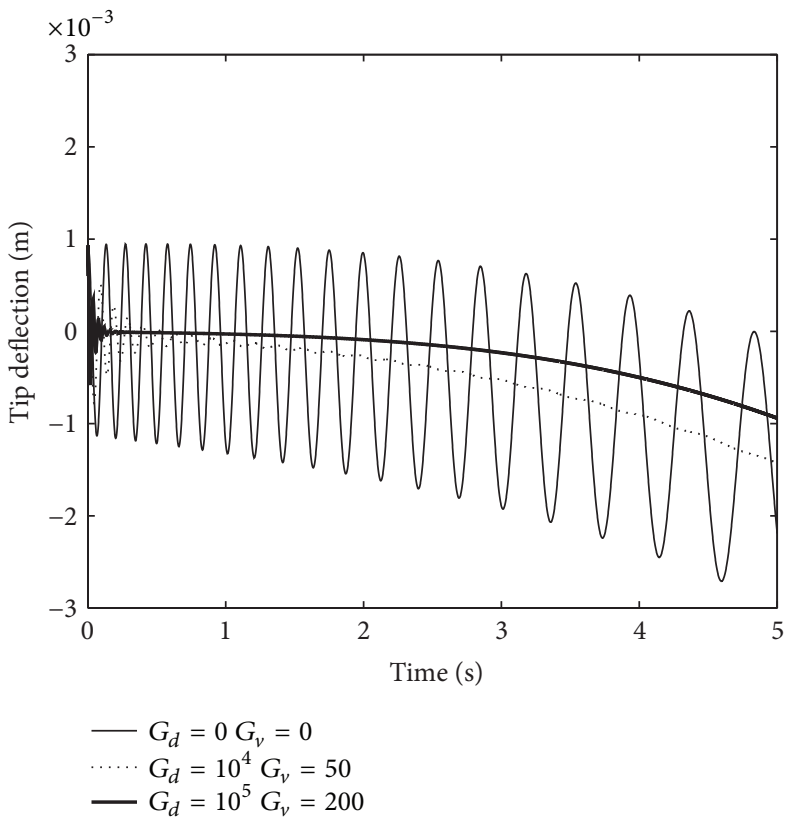

(a)

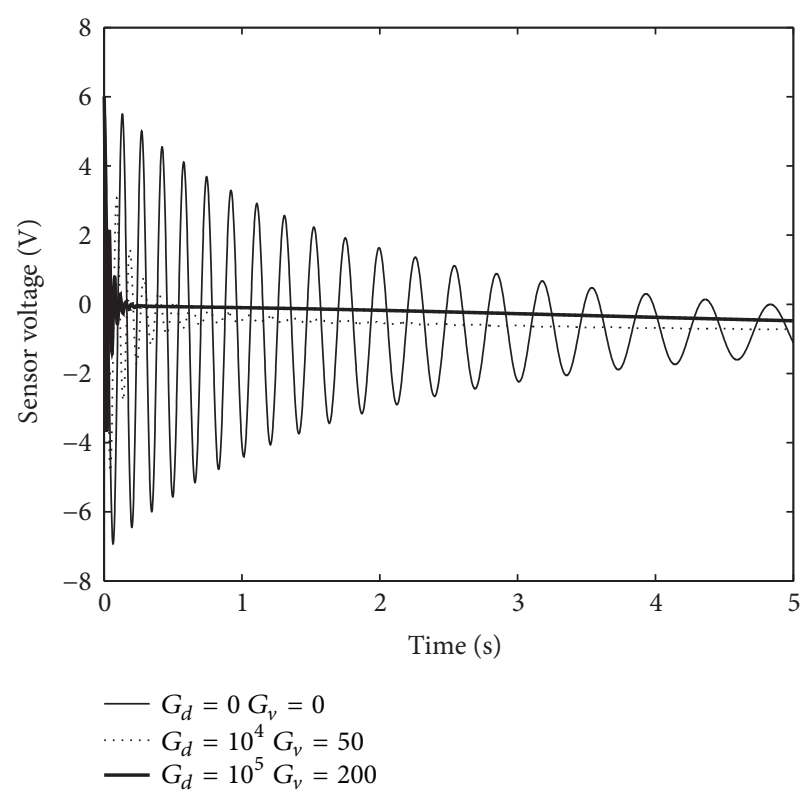

(b)

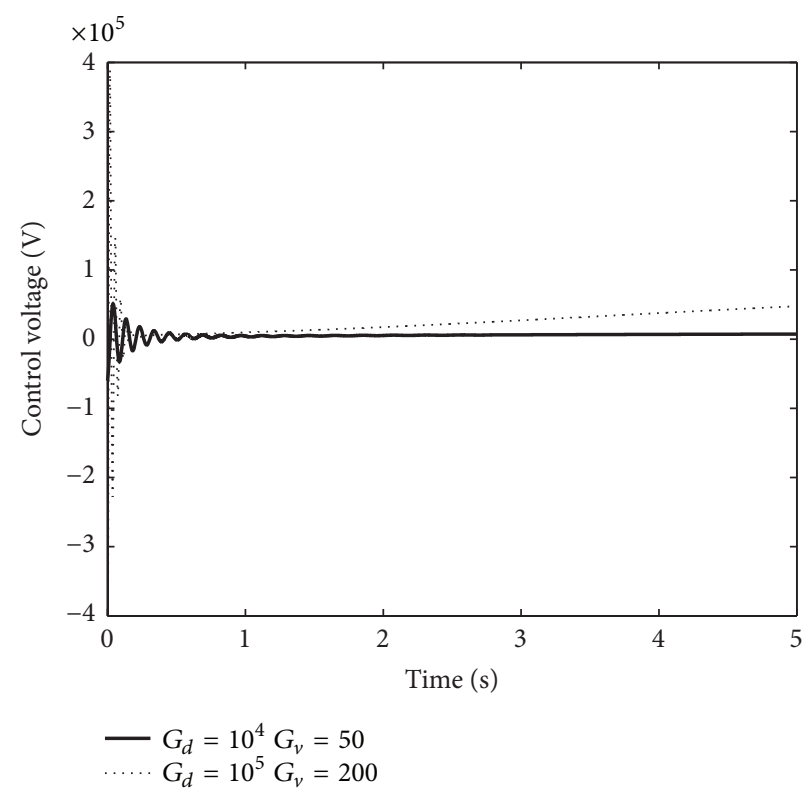

(c)

Figure 5: Tip deflection (a), sensor voltage (b), and control voltage (c) of the arm under different control gain when $L(t)=1+0.2 t, \dot{\theta}=$ $0.2 \pi \mathrm{rad} / \mathrm{s}$.

where $z_{p}$ is the $z$ coordinate of the mid-surface of the piezoelectric patch from the neutral surface of the arm. The output sensing voltage $V_{s}(t)$ can be obtained by dividing the charge by the sensor capacitance $C_{p}[26]$ :

$$
V_{s}(t)=\frac{Q_{s}(t)}{C_{p}},
$$

where

$$
C_{p}=\frac{\epsilon_{33} A_{p}}{h_{p}}, \quad A_{p}=b L_{p} .
$$

Substituting (27) into (28) we will get

$$
V_{s}(t)=\frac{e_{31} h_{p}}{\epsilon_{33} L_{p}} \int_{x_{1}(t)}^{x_{2}(t)}\left(-z_{p} \frac{\partial^{2} w}{\partial x^{2}}\right) \mathrm{d} x .
$$




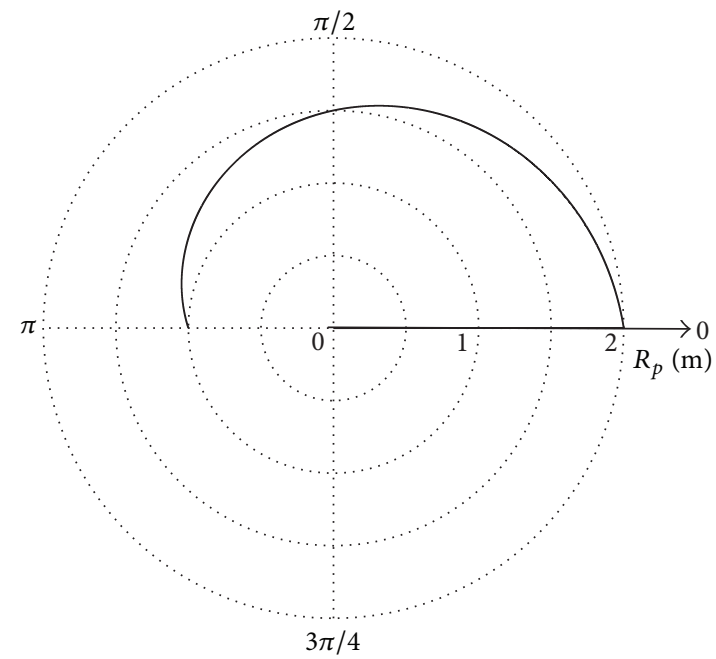

FIgURE 6: Tip trajectory of the arm when $L(t)=2-0.2 t, \dot{\theta}=0.2 \pi \mathrm{rad} / \mathrm{s}$.

The displacement and velocity feedback control law is considered and the feedback control voltage exerted on the piezoelectric patch is written as

$$
\begin{aligned}
V_{0}(t)= & -G_{d} \cdot V_{s}(t)-G_{v} \cdot \dot{V}_{s}(t) \\
= & \frac{e_{31} h_{p} z_{p}}{\epsilon_{33} L_{p}} \int_{x_{1}(t)}^{x_{2}(t)}\left[G_{d} \frac{\partial^{2} w}{\partial x^{2}}+G_{v} \frac{\mathrm{d}}{\mathrm{d} t}\left(\frac{\partial^{2} w}{\partial x^{2}}\right)\right] \mathrm{d} x \\
= & \frac{e_{31} h_{p}\left(h+h_{p}\right)}{2 \epsilon_{33} L_{p}} \\
& \times \int_{x_{1}(t)}^{x_{2}(t)}\left\{G_{d} \frac{\partial^{2} w}{\partial x^{2}}+G_{v}\left[\frac{\partial}{\partial t}\left(\frac{\partial^{2} w}{\partial x^{2}}\right)+\dot{L} \frac{\partial^{3} w}{\partial x^{3}}\right]\right\} \mathrm{d} x,
\end{aligned}
$$

where $G_{d}$ and $G_{v}$ are the displacement and velocity feedback control gains, respectively, for the piezoelectric patch. Substituting (21) into (31) results in

$$
V_{0}(t)=\left[S_{1}\right]\{\dot{q}\}^{T}+\left[S_{2}\right]\{q\}^{T},
$$

where

$$
\begin{aligned}
{\left[S_{1}\right]=\frac{e_{31} h_{p}\left(h+h_{p}\right)}{2 \epsilon_{33} L_{p} \sqrt{L}} G_{v} \int_{x_{1}}^{x_{1}+L_{p}} \frac{\mathrm{d}^{2}[\phi]}{\mathrm{d} x^{2}} \mathrm{~d} x } \\
{\left[S_{2}\right]=\frac{e_{31} h_{p}\left(h+h_{p}\right)}{2 \epsilon_{33} L_{p} \sqrt{L}} } \\
\times \int_{x_{1}}^{x_{1}+L_{p}}\left\{G_{d} \frac{\mathrm{d}^{2}[\phi]}{\mathrm{d} x^{2}}\right.
\end{aligned}
$$

$$
\begin{aligned}
+G_{v} \frac{\dot{L}}{L}\left(\frac{\mathrm{d}^{3}[\phi]}{\mathrm{d} x^{3}}(2 L-x)\right. \\
\left.\left.-\frac{5}{2} \frac{\mathrm{d}^{2}[\phi]}{\mathrm{d} x^{2}}\right)\right\} \mathrm{d} x .
\end{aligned}
$$

Substituting (32) into (25), we can obtain

$$
\begin{aligned}
& {[M]\left\{\ddot{q}^{T}\right\}+\left([C]+\left[F_{V}\right]\left[S_{1}\right]\right)\left\{\dot{q}^{T}\right\}} \\
& \quad+\left([K]+\left[F_{V}\right]\left[S_{2}\right]\right)\left\{q^{T}\right\}+\{Q\}=0 .
\end{aligned}
$$

The controlled response of the system can be gotten by solving (34).

\section{Numerical Simulation}

In order to validate the equations of motion in this paper, comparisons with the results available in the open literature are made. A uniform beam similar to that of $[11,12]$ is considered: mass per unit length $\rho A=4.015 \mathrm{~kg} / \mathrm{m}$, flexural rigidity $E I=756.65 \mathrm{Nm}^{2}$, translating velocity $v=0.3 \mathrm{~m} / \mathrm{s}$, initial length $L_{0}=1.8 \mathrm{~m}$, and initial tip deflection $w\left(L_{0}, 0\right)=$ $-0.005 \mathrm{~m}$. Tip deflections of the axially translating beam are shown in Figure 2. It is observed that the tip deflection obtained by the present work is in good agreement with that of [11]. The tip deflections of the translating and rotating beam with $L(t)=1.8+0.1 t$ and $\dot{\theta}=0.785 \mathrm{rad} / \mathrm{s}$ are plotted in Figure 3, which reveals a reasonable agreement between our work and [12].

The following numerical examples are presented to study the active control of the axially translating arm with rotatingprismatic joint. The material of the arm is steel, where $E=$ $209 \mathrm{GPa}, \rho=7800 \mathrm{~kg} / \mathrm{m}^{3}$. The piezoelectric material is PZT$5 \mathrm{H}$, where $c_{11}=49 \mathrm{GPa}, \rho_{p}=7500 \mathrm{~kg} / \mathrm{m}^{3}, e_{31}=-6.5 \mathrm{C} / \mathrm{m}^{2}$, and $\epsilon_{33}=1.3 \times 10^{-8} \mathrm{~F} / \mathrm{m}$. The dimensions of the system are 


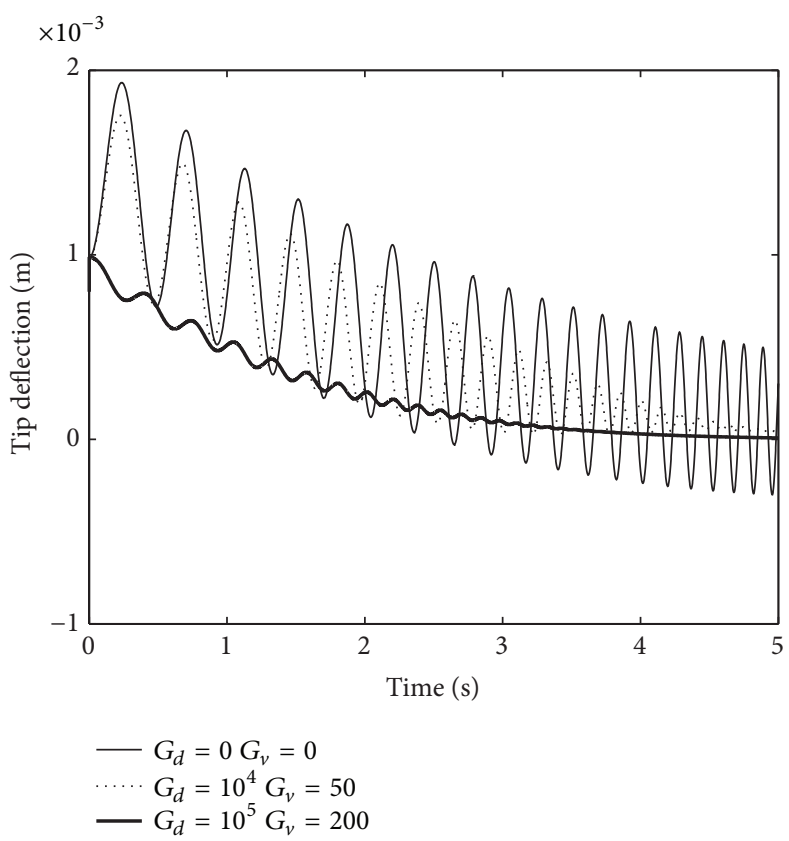

(a)

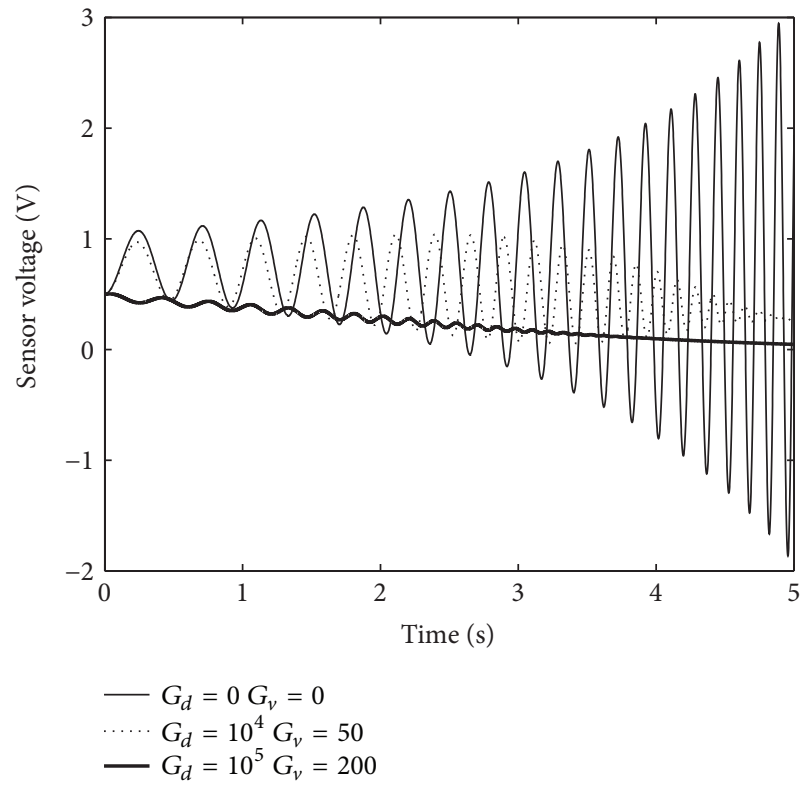

(b)

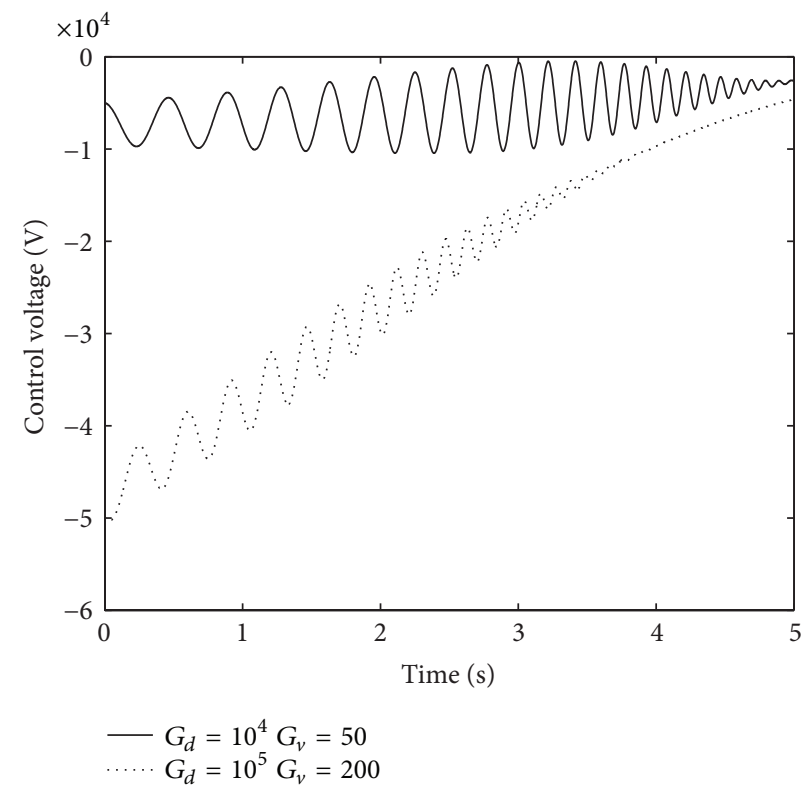

(c)

Figure 7: Tip deflection (a), sensor voltage, (b) and control voltage (c) of the arm under different control gain when $L(t)=2-0.2 t, \dot{\theta}=$ $0.2 \pi \mathrm{rad} / \mathrm{s}$. 


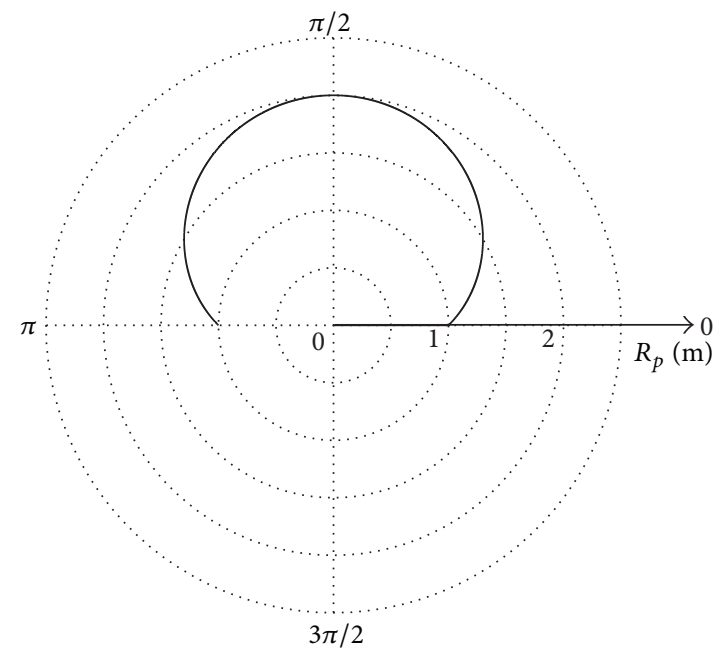

FIGURE 8: Tip trajectory of the arm when $L(t)=1+\sin (0.2 \pi t), \dot{\theta}=0.2 \pi \mathrm{rad} / \mathrm{s}$.

$L_{p}=0.5 \mathrm{~m}, b=0.2 \mathrm{~m}, h=0.01 \mathrm{~m}$, and $h_{p}=0.001 \mathrm{~m}$. The tip mass is $m_{\text {tip }}=1 \mathrm{~kg}$. The equations of motion are solved by Newmark method.

First, the arm extending at a constant axial velocity is considered. The variation in the length of the arm is expressed as $S=v t$, and the constant velocity $v=0.2 \mathrm{~m} / \mathrm{s}$. The angular velocity and acceleration are $\dot{\theta}=0.2 \pi \mathrm{rad} / \mathrm{s}$ and $\ddot{\theta}=0$, respectively. The initial conditions are $L_{0}=1 \mathrm{~m}, \theta(0)=0$, $x_{1}(0)=0, w\left(L_{0}, 0\right)=0.001 \mathrm{~m} / \mathrm{s}$, and $\dot{w}\left(L_{0}, 0\right)=0$. Figure 4 shows the tip trajectory of the arm. The tip responses of the arm and the sensor and control voltages under different control gains are plotted in Figure 5. It is observed that the self-sensing actuator can reduce the tip deflection of the arm significantly. Moreover, higher feedback control gain results in lower vibration and sensor voltage amplitudes. But the feedback control gain cannot be increased enough to a certain value beyond which the actuator will provide a larger actuating force and it will have a side effect on the vibration system [23]. It is also observed that the sensor voltage amplitude will be reduced with the extension of the arm. This is because the elastic strain of the arm section where the piezoelectric patch is placed decreases during the extension of the arm.

Next, the arm retracting at a constant axial velocity is evaluated. The variation in the length of the arm is expressed as $S=v t$, and the constant velocity $v=-0.2 \mathrm{~m} / \mathrm{s}$. We set $L_{0}=2 \mathrm{~m}, x_{1}(0)=1 \mathrm{~m}$, and other parameters are the same as previous. The tip trajectory of the arm is displayed in Figure 6. Figure 7 shows the time history of tip deflection of the arm and the sensor and control voltages under different control gains. Clearly, the self-sensing actuator can reduce the tip deflection of the arm and higher feedback control gain results in lower vibration and sensor voltage amplitudes. It is also observed that the sensor voltage amplitude will be increased with the retraction of the arm in the absence of active control. This is because the elastic strain of the arm section where the piezoelectric patch is placed increases during the retraction of the arm.
The arm travelling with harmonic length variation is also considered. The variation in the length of the arm is expressed as $S=\sin (0.2 \pi t)$. The tip trajectory of the arm is displayed in Figure 8. In this simulation, the arm experiences an extension and subsequent retraction mode. It can be observed from Figure 9(a) that the self-sensing actuator works well and increasing the control gain can enhance the performance of the controller. We can also find that the amplitude of the sensor voltage is inversely proportional to the length of the arm in the absence of active control. This is because the elastic strain of the arm section where the piezoelectric patch is placed decreases during the arm extension mode while it increases during the arm retraction mode.

\section{Conclusions}

In the present paper, active vibration control of an axially translating robot arm with rotating-prismatic joint using selfsensing actuator is investigated. The equations of the system are derived by Lagrange's equation with the assumed mode method. When the prismatic joint rotates with a uniform angular velocity, we only need to solve a linear problem. Some numerical examples are employed to evaluate the effect of the self-sensing actuator. It is found that the tip deflection of the arm can be effectively reduced by the self-sensing actuator. We can also find that the amplitude of sensor voltage is inversely proportional to the length of the arm. This is because the elastic strain of the arm section where the piezoelectric patch is placed decreases during the arm in extension mode while it increases during the arm in retraction mode. Furthermore, higher feedback control gain results in lower vibration and sensor voltage amplitudes.

In the further research work, the shear and torsional deformations in the Timoshenko beam theory should be considered. Further studies are also needed to account for nonlinear dynamic formulations involving large motion. 


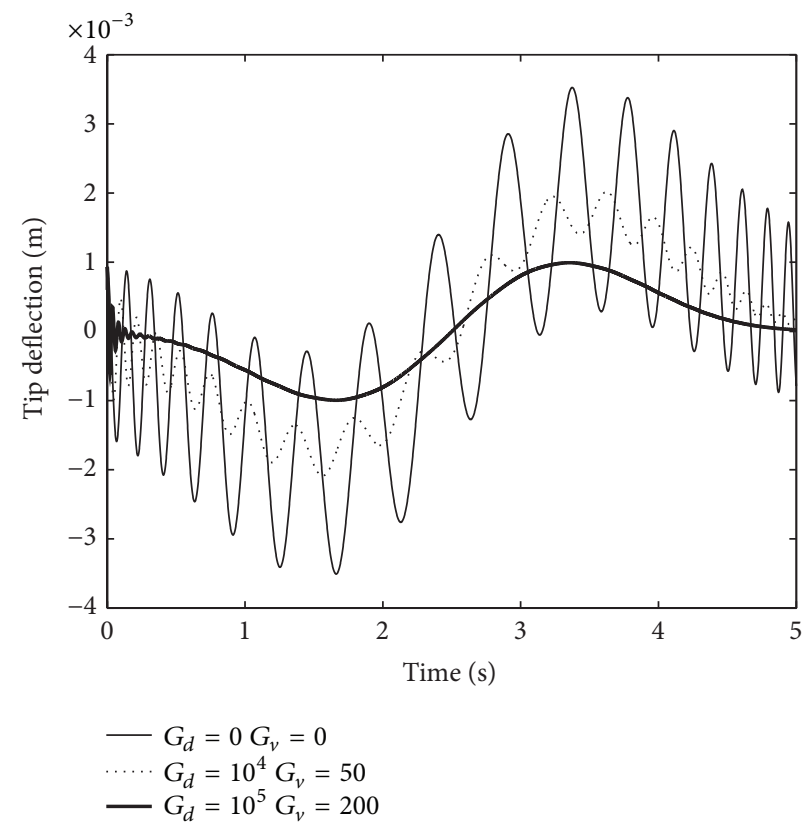

(a)

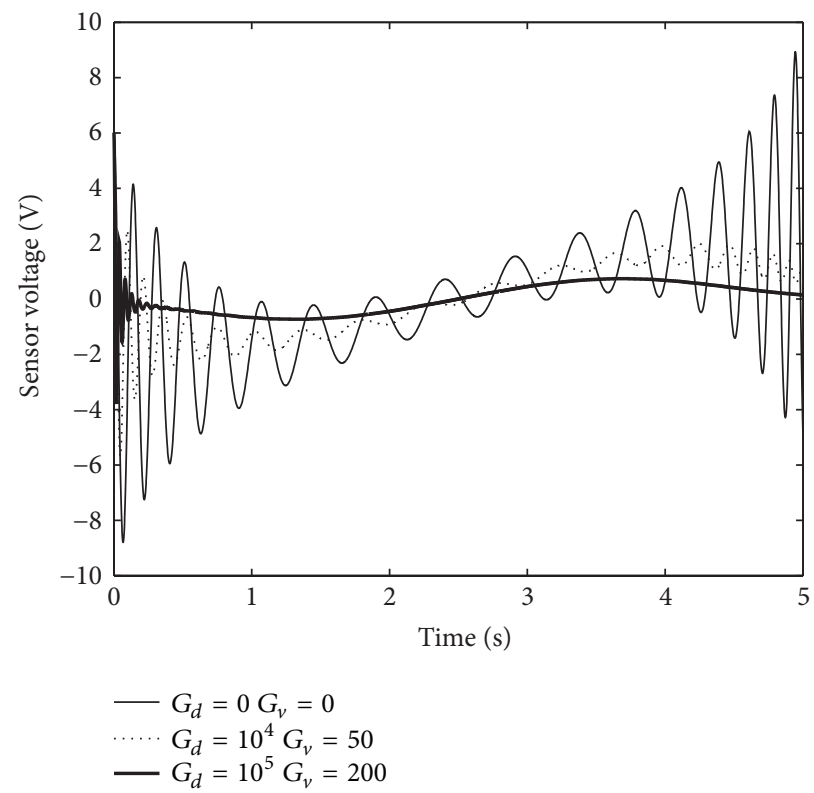

(b)

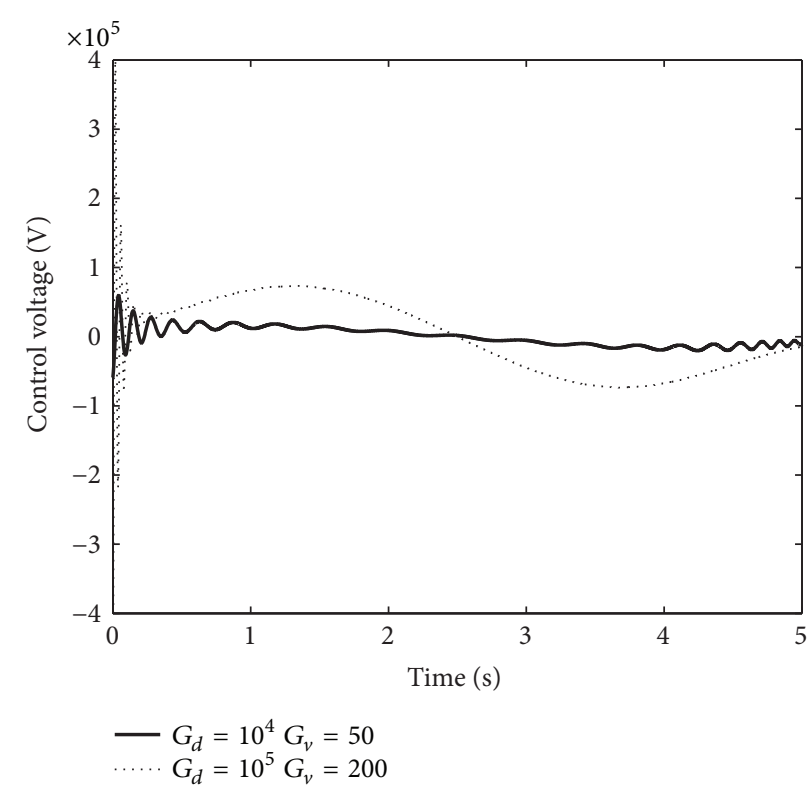

(c)

FiguRE 9: Tip deflection (a), sensor voltage, (b) and control voltage (c) of the arm under different control gain when $L(t)=1+\sin (0.2 \pi t)$, $\dot{\theta}=0.2 \pi \mathrm{rad} / \mathrm{s}$.

\section{Conflict of Interests}

The authors declare that there is no conflict of interests regarding the publication of this paper.

\section{Acknowledgment}

This work was funded by the National High Technology Research and Development Program of China (863 Program) under Project no. 2012AA041804.

\section{References}

[1] P. K. C. Wang and J. D. Wei, "Vibrations in a moving flexible robot arm," Journal of Sound and Vibration, vol. 116, no. 1, pp. 149-160, 1987.

[2] M. Stylianou and B. Tabarrok, "Finite element analysis of an axially moving beam, Part I-time integration," Journal of Sound and Vibration, vol. 178, no. 4, pp. 433-453, 1994.

[3] R.-F. Fung, P.-Y. Lu, and C.-C. Tseng, "Non-linearly dynamic modelling of an axially moving beam with a tip mass," Journal of Sound and Vibration, vol. 218, no. 4, pp. 559-571, 1998. 
[4] W. D. Zhu and J. Ni, "Energetics and stability of translating media with an arbitrarily varying length," Journal of Vibration and Acoustics, Transactions of the ASME, vol.122, no. 3, pp. 295304, 2000.

[5] L. H. Wang, Z. D. Hu, Z. Zhong, and J. W. Ju, "Hamiltonian dynamic analysis of an axially translating beam featuring timevariant velocity," Acta Mechanica, vol. 206, no. 3-4, pp. 149-161, 2009.

[6] L. H. Wang, Z. D. Hu, Z. Zhong, and J. W. Ju, "Dynamic analysis of an axially translating viscoelastic beam with an arbitrarily varying length," Acta Mechanica, vol. 214, no. 3-4, pp. 225-244, 2010.

[7] J.-R. Chang, W.-J. Lin, C.-J. Huang, and S.-T. Choi, "Vibration and stability of an axially moving Rayleigh beam," Applied Mathematical Modelling: Simulation and Computation for Engineering and Environmental Systems, vol. 34, no. 6, pp. 1482-1497, 2010.

[8] P. B. Ghaleh and S. M. Malaek, "Investigation of in-orbit disturbing loads induced on spacecraft due to elastic deploying arm," International Journal of Structural Stability and Dynamics, vol. 13, Article ID 1250081, 26 pages, 2013.

[9] P. E. Gaultier and W. L. Cleghorn, "A spatially translating and rotating beam finite element for modeling flexible manipulators," Mechanism and Machine Theory, vol. 27, no. 4, pp. 415-433, 1992.

[10] J. Yuh and T. Young, "Dynamic modeling of an axially moving beam in rotation. Simulation and experiment," Journal of Dynamic Systems, Measurement and Control, Transactions of the ASME, vol. 113, no. 1, pp. 34-40, 1991.

[11] B. O. Al-Bedoor and Y. A. Khulief, "Finite element dynamic modeling of a translating and rotating flexible link," Computer Methods in Applied Mechanics and Engineering, vol. 131, no. 1-2, pp. 173-189, 1996.

[12] B. O. Al-Bedoor and Y. A. Khulief, "Vibrational motion of an elastic beam with prismatic and revolute joints," Journal of Sound and Vibration, vol. 190, no. 2, pp. 195-206, 1996.

[13] A. M. H. Basher, "Dynamic behavior of a translating flexible beam with a prismatic joint," in Proceedings of the IEEE Southeastcon, pp. 31-38, April 2000.

[14] M. Farid and I. Salimi, "Inverse dynamics of a planar flexiblelink manipulator with revolute-prismatic joints," American Society of Mechanical Engineers, Design Engineering Division DE, vol. 111, pp. 345-350, 2001.

[15] M. Kalyoncu, "Mathematical modelling and dynamic response of a multi-straight-line path tracing flexible robot manipulator with rotating-prismatic joint," Applied Mathematical Modelling: Simulation and Computation for Engineering and Environmental Systems, vol. 32, no. 6, pp. 1087-1098, 2008.

[16] B. Sun and D. Huang, "Vibration suppression of laminated composite beams with a piezo-electric damping layer," Composite Structures, vol. 53, no. 4, pp. 437-447, 2001.

[17] S. Raja, G. Prathap, and P. K. Sinha, "Active vibration control of composite sandwich beams with piezoelectric extensionbending and shear actuators," Smart Materials and Structures, vol. 11, no. 1, pp. 63-71, 2002.

[18] L. W. Chen, C. Y. Lin, and C. C. Wang, "Dynamic stability analysis and control of a composite beam with piezoelectric layers," Composite Structures, vol. 56, no. 1, pp. 97-109, 2002.

[19] J.-C. Lin and M. H. Nien, "Adaptive control of a composite cantilever beam with piezoelectric damping-modal actuators/sensors," Composite Structures, vol. 70, no. 2, pp. 170-176, 2005.
[20] Y. R. Hu and A. Ng, "Active robust vibration control of flexible structures," Journal of Sound and Vibration, vol. 288, no. 1-2, pp. 43-56, 2005.

[21] Z.-C. Qiu, X.-M. Zhang, H.-X. Wu, and H.-H. Zhang, "Optimal placement and active vibration control for piezoelectric smart flexible cantilever plate," Journal of Sound and Vibration, vol. 301, no. 3-5, pp. 521-543, 2007.

[22] K. R. Kumar and S. Narayanan, "Active vibration control of beams with optimal placement of piezoelectric sensor/actuator pairs," Smart Materials and Structures, vol. 17, no. 5, Article ID 055008, 2008.

[23] Z. G. Song and F. M. Li, "Active aeroelastic flutter analysis and vibration control of supersonic beams using the piezoelectric actuator/sensor pairs," Smart Materials and Structures, vol. 20, no. 5, Article ID 055013, 2011.

[24] J. J. Dosch, D. J. Inman, and E. Garcia, "Self-sensing piezoelectric actuator for collocated control," Journal of Intelligent Material Systems and Structures, vol. 3, no. 1, pp. 166-185, 1992.

[25] J. M. Yellin and I. Y. Shen, "A self-sensing active constrained layer damping treatment for a Euler-Bernoulli beam," Smart Materials and Structures, vol. 5, no. 5, pp. 628-637, 1996.

[26] J. X. Gao and W. H. Liao, "Vibration analysis of simply supported beams with enhanced self-sensing active constrained layer damping treatments," Journal of Sound and Vibration, vol. 280, no. 1-2, pp. 329-357, 2005. 

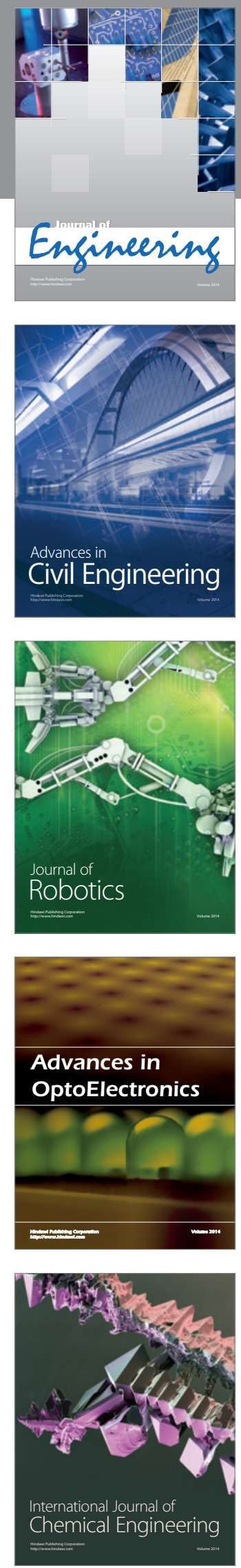

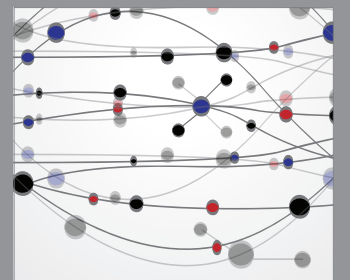

The Scientific World Journal
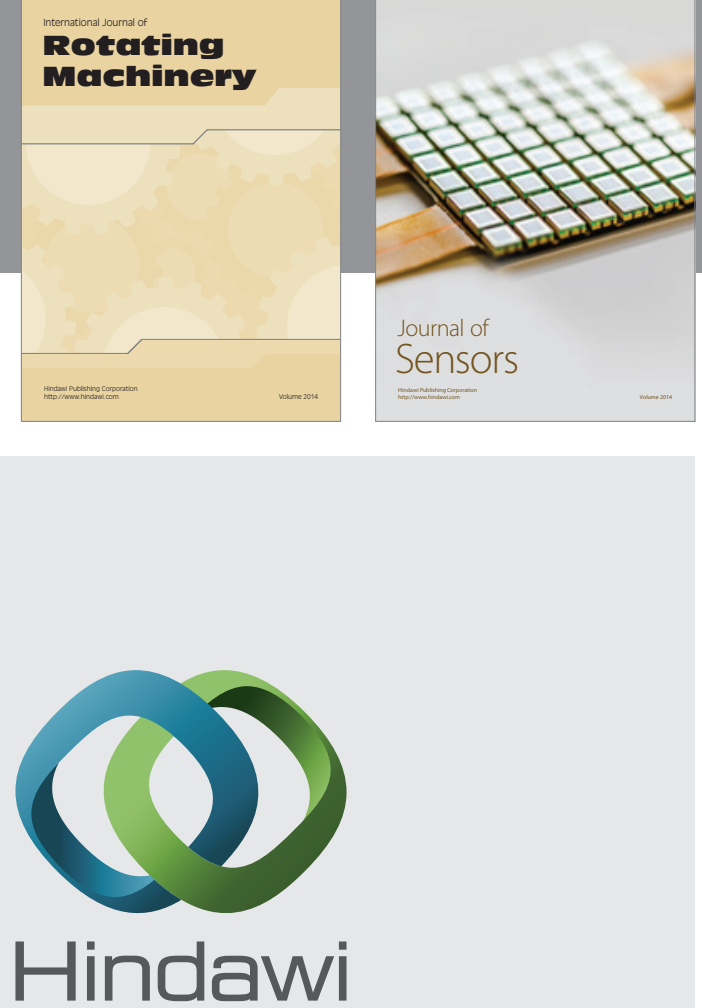

Submit your manuscripts at http://www.hindawi.com
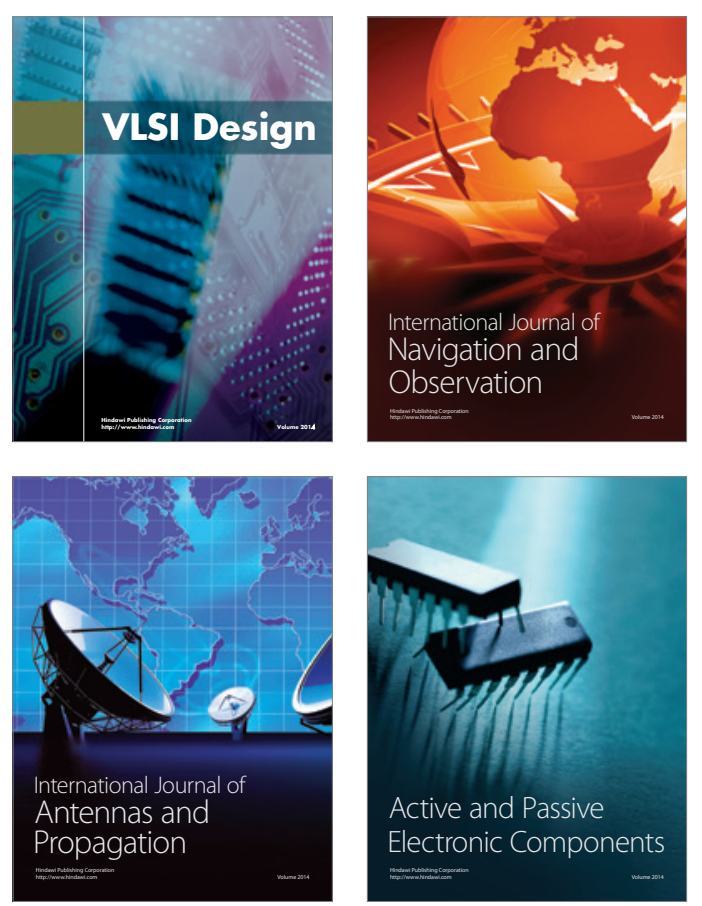
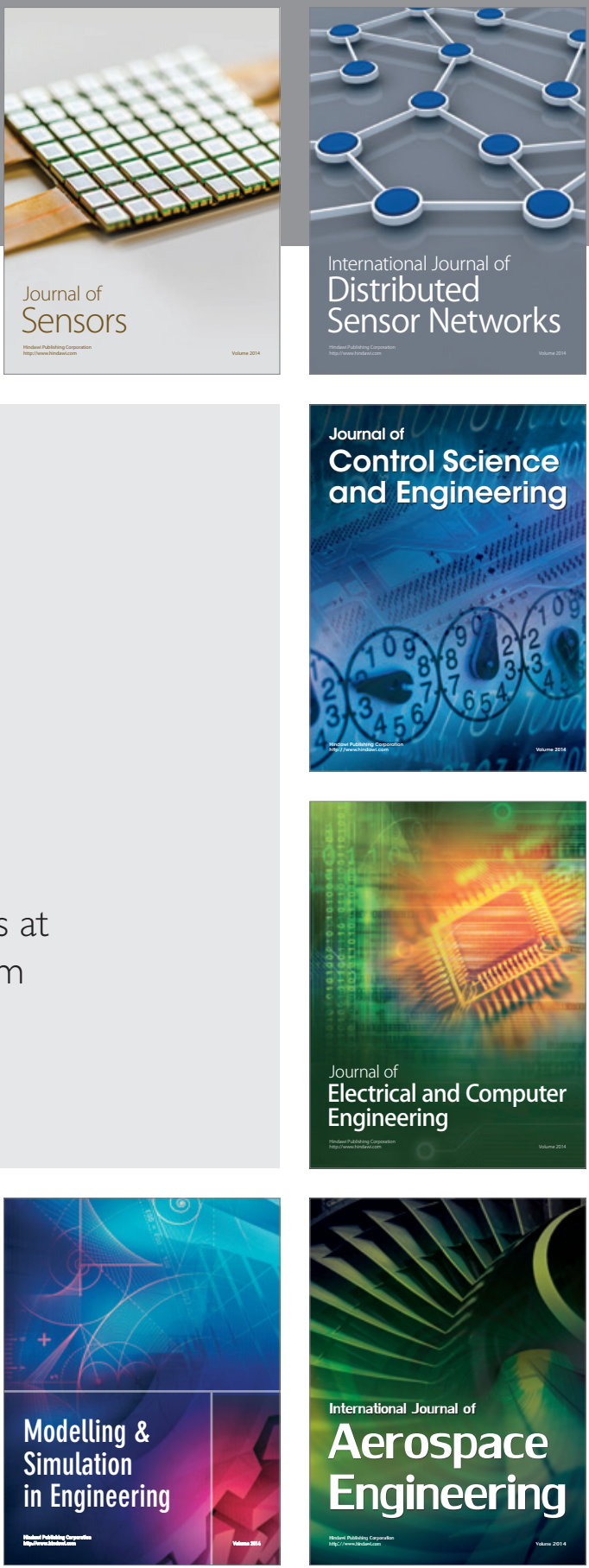

Journal of

Control Science

and Engineering
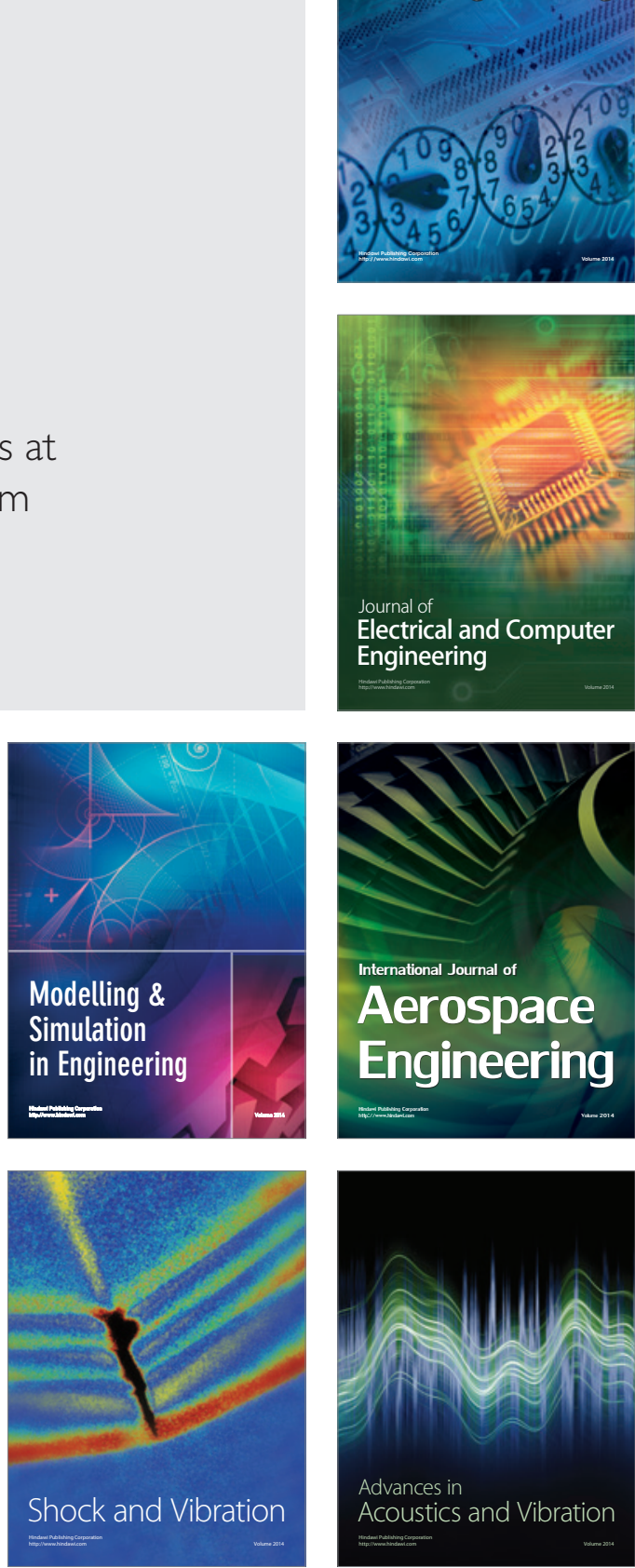